\title{
Introductory Lecture: \\ The Local and General Interstellar Medium
}

\author{
Dieter Breitschwerdt ${ }^{1,2}$ \\ 1 Max-Planck-Institut für extraterrestrische Physik, Giessenbachstrasse, D-85740 \\ Garching, Germany \\ 2 Heisenberg Fellow
}

\begin{abstract}
Important progress in the field of the Interstellar Medium (ISM) in general, and the Local Interstellar Medium (LISM) in particular, has been largely due to the recent launch of orbiting satellite missions and earth-bound observations with unprecedented sensitivity and high spectral or spatial resolution. However, the interpretation of a wealth of new data is still based to a great extent on models that were designed for the previous generation of observations. Thus there remains a clear gap between our theoretical understanding of the basic physics of the ISM and the detailed information available. In such a situation it seems appropriate to focus on some key observations and discuss their implications for modelling.
\end{abstract}

\section{Introduction}

This opening lecture was originally supposed to be given by Lyman Spitzer. On behalf of the Scientific Organizing Committee J. Trümper and myself wrote an invitation letter to him, which remained unanswered. A couple of weeks later, we learned of his sudden death, which means a great loss for his many friends in the astronomical community and especially among students of the ISM. It was clear that just a few weeks before the conference, we would not be able to find an adequate replacement.

In this article I will content myself to give a short overview of observational and theoretical progress with no attempt of completeness and doing justice to all the people who have been so heavily involved in this field over the past years. For brevity, I will confine myself to some key observations and models. In a couple of years time it may well appear in hindsight, that other observations and models will be more relevant to our understanding. But then progress rarely evolves in straight paths, and therefore any observation that is in conflict with our theoretical understanding now deserves to be discussed in this context.

\section{Brief Overview of the General Interstellar Medium}

The subject of the ISM is nowadays a classical field of astronomy. Nevertheless, as will be discussed in more detail below, there have always been surprises 
even until to date, that keep the field young and prevent researchers from falling into dogmatism.

Historically, stationary absorption lines of ionized calcium in a spectroscopic binary have been studied already at the beginning of the century (Hartmann, 1904), but it was not until the early 30's when it became clear that these lines were of interstellar and not circumstellar origin (Plaskett and Pearce, 1933). Previous work on extinction of star light lead to the concept of a pervasive gaseous and dusty medium concentrated in the disk of the Galaxy. However, kinematical information on absorption lines showed that the radial velocities differed substantially in different regions of the Galaxy (Adams, 1948), and therefore the distribution of gas must be far from uniform. A global mapping of the gas became available by $21 \mathrm{~cm}$ observations, following an earlier theoretical prediction of the hyperfine structure transition of $\mathrm{HI}$ by van de Hulst (1945). It was clear that a possible source of energy for the interstellar gas were embedded stars. Strömgren (1939) showed that early-type stars should have rather sharp $\mathrm{HI} / \mathrm{HI}$ boundaries, called ionization fronts. Since the interior is kept ionized by the stellar photon field, the "Strömgren sphere" will undergo dynamical expansion due to its overpressure and drive a shock wave into the ISM. Although there is an energy input of more than $10^{48} \mathrm{~s}^{-1}$ ionizing photons from an O9 star, say, the energy conversion factor $\epsilon$ of Lyman continuum photons into kinetic energy of the ISM can be estimated to be very small: $\epsilon \leq 10^{-2}$; most of the energy is radiated away and other sources of energy input had to be sought. The first attempts to explain the nonuniform distribution of interstellar matter into distinct "phases" that would be stable against pressure perturbations were undertaken by Pikel'ner (1967) and Field, Goldsmith and Habing (1969). The latter envisaged ionization of the ISM by low energy cosmic rays to be the main source of energy input, whereas energy losses were due to collisional excitation and subsequent line cooling. This so-called 2-phase model explained the coexistence of a cold neutral phase $\left(T \sim 30 \mathrm{~K}, n \sim 100 \mathrm{~cm}^{-3}\right)$ with a warm and weakly ionized phase $\left(T \sim 10^{4} \mathrm{~K}, n \sim 0.3 \mathrm{~cm}^{-3}\right)$.

A major thrust in our understanding of the ISM came with the launch of COPERNICUS in 1972, which was inspired and promoted by Lyman Spitzer. The detection of the ubiquitous OVI absorption line and a careful kinematic analysis, that separated the absorbing regions from the circumstellar medium, lead to the establishment of a widespread hot interstellar medium (Jenkins and Meloy, 1974; York, 1974) with $5.4 \leq \log \mathrm{T} \leq 6.3$. Already a few years before, in a pioneering rocket experiment Bowyer et al. (1968) had revealed the existence of diffuse soft X-ray emission in the energy range below $1 \mathrm{keV}$. It could be decomposed into an extragalactic and what they called at the time an "anomalous" component, which they speculated might be of Galactic origin (see also Bowyer, this volume). Such an emission source had been suggested long ago by Spitzer (1956), who argued for a hot $\left(T \sim 10^{6} \mathrm{~K}\right)$ Galactic corona, in order to provide a pressure confining medium for the then observed 
high velocity clouds. It became clear however, that the hot medium observed with COPERNICUS could not reproduce the spectral characteristics of the soft X-ray background (SXRB), so that it seemed as if both observations were sampling different gas.

Since the prime energy sources in the ISM were known by then to be Type II supernovae (SNe), the question to be answered remained how to create a pervasive hot medium. Cox and Smith (1974) argued that according to Monte-Carlo simulations of randomly occurring SNe in a homogeneous disk, which they followed until the dispersal of the remnants in the ISM, a tunnel network of hot bubbles $\left(T \sim 10^{6} \mathrm{~K}, n \leq 10^{-2} \mathrm{~cm}^{-3}\right)$ with a volume filling factor of $f_{\mathrm{HIM}}^{\mathrm{V}} \sim 0.5$ could be achieved for a Galactic SN rate of $1 / 50 \mathrm{yr}^{-1}$ and an average explosion energy of $\mathrm{E}_{\mathrm{SN}} \sim 4 \times 10^{50} \mathrm{erg}$ in an ambient medium of density $n_{\mathrm{ISM}} \sim 1 \mathrm{~cm}^{-3}$. In a seminal paper, McKee and Ostriker (1977) undertook the task to combine all that was known at the time into a consistent picture. It was a bold attempt and a brilliant piece of work to devise a theory for the ISM, which owing to its complexity has mostly been and still is dissected into more or less isolated problems which can then be tackled. They were lead by the compelling idea that SN explosions in an inhomogeneous cloudy ISM should result in a time-asymptotic picture as it is observed presently. The net result is a three-component ISM in the Galactic disk, consisting of a cold neutral medium (CNM) coated by warm neutral clouds (WNM), with their ionized surfaces representing the warm ionized medium (WIM). Most of the volume is filled with hot intercloud medium (HIM) with a filling factor as large as $f_{\mathrm{HIM}}^{\mathrm{V}} \approx 0.7-0.8$. Global mass and energy balance between cloud evaporation and dense shell formation and SN heating and radiative cooling, respectively, provides the observed density and temperature structure (see also for more details McKee, this volume).

While this model has certainly outlived all its competitors and is probably more often quoted than any other ISM paper, we have to ask ourselves, whether it is still correct in general and with respect to the Local Bubble in particular. My answer with respect to the general ISM would be yes and no. The basic ingredients I believe are correct: SNe are the main energy source of the ISM and they are responsible for producing hot and X-ray emitting plasma all over the disk and halo by interaction with an inhomogeneous substrate; however, the high value of $f_{\mathrm{HIM}}^{\mathrm{V}}$ has been challenged by observations in the 80's. H I observations in the Milky Way (Heiles, 1980) and in nearby galaxies (Brinks and Shane, 1984) have shown the existence of supershells and $\mathrm{HI}$ holes, respectively, which point towards a much smaller filling factor of $f_{\mathrm{HIM}}^{\mathrm{V}} \sim 0.2$, assuming an anticorrelation between hot and neutral gas. Only 100 pc away is the Loop I superbubble, which has a radius of about 150 pc and which has been filled with hot X-ray emitting plasma, as observed by ROSAT (Egger and Aschenbach, 1995), generated by about $40 \mathrm{SNe}$ in concert. At present it seems that probably half of the SNe occur in a temporally and spatially correlated fashion. Thus highly overpressured regions 
will be expanding preferentially perpendicular to the galactic plane and deposit mass, momentum and energy into the lower halo. This may result in a "clustered" galactic fountain type flow as described in the so-called "chimney model" (Ikeuchi, 1988; Norman and Ikeuchi, 1989; see also Ikeuchi, this volume) circulating matter between the disk and the halo and/or a galactic wind (Breitschwerdt et al., 1991; 1993). In any case, $f_{\mathrm{HIM}}^{\mathrm{V}}$ in the disk will be lowered. Global pressure equilibrium between phases is not satisfied, at least locally, because a large pressure imbalance of a factor of eight between the hot component and the Local Cloud has been found from EUV cloud shadowing (Berghöfer et al., this volume). Further, pulsar dispersion measurements have revealed the existence of diffuse ionized gas (DIG), occupying a volume fraction of 0.2 with an exponential vertical scale height of $z \sim 1 \mathrm{kpc}$ (Reynolds, 1991). No current ISM model can explain the distribution of the DIG, its characteristic line ratios, and above all how it is kept ionized.

Although the volume filling factor of the HIM has been reduced to some extent, it still appears that half of the ISM is filled by a hot and tenuous plasma. Thus it is not unusual that our solar system is also located in such a medium. It is suggestive to assume that our local environment will therefore be an ideal laboratory for investigating the hot phase of the ISM. In the remainder of this paper, I will address this problem in more detail. Before closing this section, I would like to remind the reader that the ISM is not merely the matter between the stars. In physical terms, it is more adequately described by the components that dominate its energetics. It is well-known that, at least locally, a global energy equipartition does exist between the thermal and kinetic energy density of the plasma and the energy density of the magnetic field and the cosmic rays, which are all about $1 \mathrm{eV} / \mathrm{cm}^{3}$. Rather than being a fortuitous result, this indicates that a strong dynamical coupling between all of these ISM components is present. From a theorist's point of view this is not surprising, because in the ISM the magnetic field is well frozen into the plasma, and the cosmic rays can strongly interact by scattering off the random component of the field, thus imparting momentum and energy to the plasma while propagating through the ISM. Any theory of the ISM should therefore include the momentum and energy exchange between plasma, magnetic field and cosmic rays in the disk and the galactic halo (Breitschwerdt, 1994).

\section{The Local Interstellar Medium (LISM)}

The LISM has been the subject of a number of reviews (Cox and Reynolds, 1987; McCammon and Sanders, 1990; Breitschwerdt, 1996) and I will therefore restrict myself to outline some key observations and models, which I consider as important for future progress.

Historically, the term Local Hot Bubble (LHB) resulted from the observational fact that an anticorrelation between soft X-ray emitting plasma and 
HI exists on large angular scales. The constancy in intensity of different ultrasoft X-ray bands ( $E \leq 0.3 \mathrm{keV}$ ) with largely different absorption cross sections (unity optical depth corresponding to a column density as small as $N(\mathrm{H}) \sim 10^{19} \mathrm{~cm}^{-2}$ ) could only be understood, if the region surrounding the solar system was essentially devoid of HI out to distances of $100 \mathrm{pc}$ with considerable variation in galactic latitude. Owing to the high $N(\mathrm{H})$ in the Galactic plane (at $b^{I I}=0^{\circ}$ ) it was evident that there had to be a local emission component. Thus the term LHB was "in the air" and appeared almost simultaneously in the literature by a Japanese and the Wisconsin group (Tanaka and Bleeker, 1977; Sanders et al., 1977). Such a displacement of the cold gas by X-ray emitting hot gas was suspected by McKee and Ostriker (1977) to be the result of a local SN remnant (SNR). Following this idea, Cox and Anderson (1982) were modelling the expansion of a single blast wave of energy $5 \times 10^{50} \mathrm{erg}$ propagating into a low density $\left(n_{0}=4 \times 10^{-3} \mathrm{~cm}^{-3}\right)$ ambient medium with non-negligible pressure. Due to the fixed radial scale, such a model is heavily constrained, with the explosion having occurred about $10^{5}$ yrs ago. Alternatively, it has been argued that the LHB is the relic of an old superbubble (Innes and Hartquist, 1984) which has been excavated $4 \times 10^{6}$ yrs ago, by a collective SN energy of $10^{52} \mathrm{erg}$, where the ambient medium density is $n_{0}=1 \mathrm{~cm}^{-3}$. Since the bubble has been subject to heavy radiative losses, the spectrum becomes progressively harder with time, so that M-bands emission $(0.5-1 \mathrm{keV})$ can be explained as well in contrast to the young SNR model. Although not conclusive, cosmic ray data from meteorites and Antarctic ice cores show that the cosmic ray flux seems to have been remarkably constant over the last $10^{9}$ yrs with two peaks $3.5 \times 10^{4}$ and $7 \times 10^{4}$ years ago (Sonett et al., 1987; see also Morfill, this volume).

Observations tell us, that emission in the M-bands is more isotropic than in the softer bands and that roughly $15 \%$ can be accounted for by stellar disk sources (Schmitt and Snowden, 1990). It is still unclear at present how much of the M-bands emission is produced in the Local Bubble and what fraction comes from the galactic halo and from extragalactic sources. An upper limit at present seems to be about $20 \%$ for a local contribution (Snowden et al., 1993). This value is hard to achieve in the LHB model (e.g. Snowden et al., 1990 ), in which originally all of the emission below $0.3 \mathrm{keV}$ was produced in an asymmetric cavity filled with a hot $\left(T=10^{6} \mathrm{~K}\right)$ and tenuous $\left(n_{b}=\right.$ $4.7 \times 10^{-3} \mathrm{~cm}^{-3}$ ) plasma radiating in collisional ionization equilibrium (CIE). A direct measurement of foreground M-bands emission has become possible with the ROSAT PSPC (Trümper, 1983) owing to its much higher sensitivity and angular resolution than any previous instrument. In so-called shadowing experiments, the SXRB is used to probe absorbing material between the source and the observer. Taking high column density $\left(N(\mathrm{H}) \geq 10^{21} \mathrm{~cm}^{-2}\right)$ absorbers like the molecular cloud MBM12, which is located inside the Local Bubble (distance $D \sim 60-70$ pc; cf. Snowden et al., 1993), M-bands emission from behind can be efficiently blocked off. An upper limit of 0.3 for the 
ratio of on- to off-source intensity has been derived (Snowden et al., 1993). Unfortunately, the number of such clouds inside the Local Bubble is too small to draw any far reaching conclusions. For absorbers at larger distances it is much more complicated to disentangle the various contributions of emission and absorption. Nonetheless, a careful systematic investigation would be most valuable.

At this point, it should be emphasized, that we have no direct evidence for the physical state of the local plasma. In other words, we cannot measure its density, temperature and pressure. Instead, within the LHB model, the temperature is commonly inferred by a broad band spectral fitting procedure using a Raymond and Smith (1977) type plasma code (for details see Böhringer, this volume) for a gas in CIE at energies below $0.3 \mathrm{keV}$. Since only the emission measure is fixed by the spectral intensity, the density is derived once the pathlength of the emitting volume has been chosen. This is usually done under the assumption that the hot gas fills the volume devoid of HI completely.

A serious problem in the framework of the LHB model is the discrepancy between the inferred pressure of $P / k_{B} \approx 10000 \mathrm{~cm}^{-3} \mathrm{~K}$, where $k_{B}$ is Boltzmann's constant, and that of the Local Cloud, for which $P / k_{B} \approx$ $1700-2600 \mathrm{~cm}^{-3} \mathrm{~K}$ (Lallement, this volume). It is hard to imagine how such a cloud can survive being crushed within a relatively short time scale. Moreover, there is observational evidence of other $\mathrm{HI}$ clouds and filaments within the Local Bubble, e.g. the "finger" filament (Kerp et al., 1993).

The most promising way, we can reasonably well define the plasma state in the future seems to be the following:

- We need high resolution spectroscopy in soft X-rays and EUV in order to identify individual lines.

- Pulsars with extremely low dispersion measures and known parallaxes can provide the average electron density $\left\langle n_{e}\right\rangle$ in the Local Bubble, independently of spectral fitting.

Since we get only information on $\left\langle n_{e}\right\rangle$, the distribution of ionized matter in the Local Bubble may be far from homogeneous. It is conceivable that an intermixture of hot plasma and clouds surrounded by WIM, like in the McKee and Ostriker (1977) model, provides the bulk of the dispersion. However, taking their mean cloud radius of $2.1 \mathrm{pc}$ (from their Table 1), whith the outermost $0.5 \mathrm{pc}$ in radius corresponding to the WIM, we also sample on average $N(\mathrm{H}) \sim 3 \times 10^{20} \mathrm{~cm}^{-2}$ of CNM column density per cloud. Given a volume filling factor of $f_{\mathrm{WIM}}^{\mathrm{V}}=0.23$ one expects to intercept about 30 clouds within any line of sight extending out to a radius of $100 \mathrm{pc}$. Therefore an average $N(\mathrm{H}) \sim 9 \times 10^{21} \mathrm{~cm}^{-2}$ should be detectable in soft X-rays. Moreover, $N(\mathrm{H})$ should increase almost linearly with distance. But observations (Fruscione et al., 1994) show that this is not the case for most sight lines; instead a shallow gradient is seen in most directions, followed by a steep increase at about $100 \mathrm{pc}$. Alternatively, making the clouds smaller and thus reducing their $\mathrm{HI}$ 
content but retaining their HII will also decrease their life time with respect to destructive mechanisms like evaporation. Hence, although we do not constrain the distribution of the medium by pulsar dispersion measures, we are restricted by the HI content of intermixed clouds and their average life time.

An intrinsic problem with nearby pulsar dispersion measures is statistics. There are only very few pulsars in the solar vicinity. PSR $0950+08$ has a parallactic distance of 125 pc (Gwinn et al., 1986). It is located in a direction where the Local Bubble extension varies considerably, i.e. between 37 and 347 pc (Fruscione et al., 1994); alternatively, the large path length could also be due to a void of Hi outside the Local Bubble. PSR J01081431 has the lowest known dispersion measure $D M=1.83 \mathrm{~cm}^{-3} \mathrm{pc}$ (Tauris et al., 1994). The predicted distance, using a model for the Galactic electron distribution (Taylor and Cordes, 1993), is about $90 \mathrm{pc}$, corresponding to $\left\langle n_{e}\right\rangle \approx 2.2 \times 10^{-2} \mathrm{~cm}^{-3}$. Unfortunately the pulsar is located in a direction where the ROSAT count rates are fairly low and the Local Bubble boundary is closer than average to the Sun. Some time ago, we have predicted the distance of the binary millisecond pulsar J0437-4715 (DM $\left.=2.65 \mathrm{~cm}^{-3} \mathrm{pc}\right)$ to be about $110 \mathrm{pc}$ on the basis of a non-CIE Local Bubble model with an average density of $n \sim 2.4 \times 10^{-2} \mathrm{~cm}^{-3}$ assuming that it is located inside the Local Bubble (Breitschwerdt and Schmutzler, 1994). Using the model of Taylor and Cordes (1993), the inferred distance is 140 pc. Due to the fact that we do not know whether these pulsars are inside the Local Bubble, there is no conclusive answer to the average density yet. Also the peculiar line of sight towards $\beta \mathrm{CMa}$, which is largely filled with HiI gas reaching out to a distance of $200 \mathrm{pc}$, with $\left\langle n_{e}\right\rangle \approx 2 \times 10^{-2} \mathrm{~cm}^{-3}$ and a very low temperature $T \leq 5 \times 10^{4} \mathrm{~K}$ adds to the mystery of the plasma state in the Local Bubble.

With high spectral resolution in the soft X-ray bands, we will be able to fix a unique temperature to the plasma, if it is in CIE. Alternatively, if it is not in CIE, we need more sophisticated models in which the dynamical and thermal evolution is coupled (e.g. Breitschwerdt and Schmutzler, 1994).

Both the DXS and EUVE experiments (see Sanders and Vallerga, respectively, this volume) were a major step in this direction. EUVE was not designed for observing diffuse emission in the energy range $70-760 \AA$ and DXS $(0.15 \leq E \leq 0.284 \mathrm{keV})$ has faced some problems during data collection and calibration. Nevertheless, some tentative conclusions can be drawn. Due to their low mean free path EUV photons are an excellent probe for the very LISM. Jelinsky et al. (1995) have given upper limits on the diffuse EUV flux. Bearing in mind that a CIE plasma at $10^{6} \mathrm{~K}$ like in the LHB model should exhibit a plethora of EUV emission lines, it is remarkable that the only lines detected were HeI and HeII, with intensities that are consistent with geocoronal and/or interplanetary scattering of solar radiation. The plasma emission measure is a factor of 5 to 10 below of what is expected from a CIE plasma in the range $10^{5.7} \leq T \leq 10^{6.4} \mathrm{~K}$. The spectral resolution of the DXS Bragg crystal spectrometers is $10-25 \mathrm{eV}$ while the spatial resolution is only $\sim 15^{\circ}$. 
One of the most important results has been the detection of emission lines, which is a direct evidence for diffuse emission of a thermal plasma (Sanders, 1993). Again, it is not possible to fit the spectrum by a CIE plasma with normal or depleted abundances.

Dropping the assumption of CIE, gives rise to a new class of models that show some promising features. There are essentially two extreme possibilities. The first is fast heating (e.g. by a shock wave) of interstellar plasma, which leads to an underionization of the gas; in this case delayed ionization causes the heavy species to have lower ionization stages than a plasma at the same temperature in CIE. Such a case has been considered e.g. by Cox and Anderson (1982), who followed the expansion of a young SNR into an ambient medium with non-negligible pressure. The second possibility is fast adiabatic expansion of a plasma that had been heated up during its previous history to high temperatures $\left(T \geq 10^{6} \mathrm{~K}\right)$, at which the assumption of CIE is fairly good; in this case delayed recombination essentially retains the high ionization stages of the expanding gas and the emission spectrum is similar to the original CIE plasma. We have considered this process in detail in connexion with a galactic outflow expanding away from the disk, and also for the origin of the Local Bubble (Breitschwerdt and Schmutzler, 1994; 1997). In the latter case, we assumed that the Local Bubble is the relic of an old superbubble, which was created by about 20 early type stars injecting about $10^{52} \mathrm{erg}$ into a dense molecular cloud $\left(n_{0} \sim 10^{4} \mathrm{~cm}^{-3}\right)$. After about $10^{7} \mathrm{yr}$, the superbubble breaks out of the cloud and undergoes fast adiabatic expansion, with the last SN having occurred roughly $1.5 \times 10^{6} \mathrm{yr}$ ago. The characteristic features of this model are:

- The emission spectrum is characterized by recombination and line emission and is similar to a $10^{6} \mathrm{~K}$ CIE spectrum; however it is deficient in EUV lines due to the low kinetic temperature and is therefore consistent with the EUVE observations.

- There is a local contribution to the M-bands (although quantitatively this depends on the initial model)

- The plasma kinetic temperature at present is only about $4.2 \times 10^{4} \mathrm{~K}$ and the density is $2.4 \times 10^{-2} \mathrm{~cm}^{-3}$. Thus the thermal pressure is $P / k_{B} \approx$ $2000 \mathrm{~cm}^{-3} \mathrm{~K}$ and therefore very close to that of the Local Cloud. The density is in agreement with the one inferred from the dispersion measure of the pulsar PSR 0950+08.

It is important to emphasize that the dynamical and thermal evolution of the plasma are intrinsically coupled and hence a self-consistent solution has to be calculated. There is no modified cooling function that can be plugged into the dynamical equations, since the dynamics changes the thermal path of the gas and vice versa.

The model presented above is by no means unique; it can explain all present observations, but might fail for future ones. The natural approach for non-CIE plasma evolution is to systematically narrow down the number 
of possible initial conditions by observations, and thus eventually find a model that comes reasonably close to reality.

\section{Beyond the Local Bubble}

One of the key experiments concerning the Local Bubble and the SXRB are the shadowing experiments already mentioned in the previous section. Among the first targets was a deep pointed observation of the Draco nebula (Burrows and Mendenhall, 1991; Snowden et al., 1991), that revealed that about half of the C-band emission originates behind Draco (minimum distance $D \sim 300 \mathrm{pc}$ ), i.e. way beyond the Local Bubble. Although the protagonists of the LHB or displacement models have argued that this does not make any serious difference (e.g. Snowden, 1996; see also Cox, this volume), because it would only change the shape of the LHB somewhat, there remains the fact to be explained how C-band emission outside the LHB is generated in the absence of any distinct superbubbles. Even shadows much further away $(D \geq 1.5 \mathrm{kpc})$, cast by Complex M of high velocity clouds, reveals SXR emission (Herbstmeier et al., 1995). Recently, the HI information of the northern sky has been considerably improved by the new Leiden-Dwingeloo Survey.

Also C-band emission from the halo of the edge-on spiral NGC 4631 has been reported (Wang et al., 1995), and there does not seem to be much doubt of soft X-ray emission also from the Galactic halo. How does it come about? While the HIM envisaged by McKee and Ostriker (1977) with a temperature of $T \sim 10^{5.7}$ is too cool to produce significant C-band emission, their Galactic corona with $T \geq 10^{6} \mathrm{~K}$ and a pressure scale height of $h=5\left(T / 10^{6} \mathrm{~K}\right) \mathrm{kpc}$ (due to the low density of cooling clouds and energy injection from halo $\mathrm{SNe}$ ) should do so. However, such a halo is difficult to keep in hydrostatic equilibrium for various reasons. Most simply, the cooling time $\tau_{\text {cool }}$ must be much larger than the dynamical relaxation time, i.e. $P_{h}^{3 / 2} /\left(q \rho_{h}^{5 / 2}\right) \gg c_{s} / g_{z}$ or $c_{s}^{2} \gg \rho_{h} q / g_{z}$ where Kahn's (1976) $T^{-1 / 2}$-cooling-law has been applied; here $P_{h}, \rho_{h}, c_{s}$ and $g_{z}$ denote the halo pressure and density, the speed of sound, the $z$-component of the gravitational acceleration, respectively and $q=4 \times 10^{32} \mathrm{~cm}^{6} \mathrm{~g}^{-1} \mathrm{~s}^{-4}$ is a parameter (see Kahn, this volume). With $\rho_{h}=$ $2 \times 10^{-27} \mathrm{~g} / \mathrm{cm}^{3}, c_{s}=130 \mathrm{~km} / \mathrm{s}$ and $g_{z}=10^{-8} \mathrm{~cm} / \mathrm{s}^{2}$, the above condition is hard to fulfill.

Moreover, it is very likely that cosmic rays with a comparable energy density but an essentially infinite pressure scale height will couple to the plasma through the resonant generation of MHD waves and thus drive a fraction of the plasma out to infinity in the form of a supersonic wind (Breitschwerdt et al., 1991; 1993). The rest has to rain down in a fountain (Bregman, 1980; Kahn 1981; Avillez et al., this volume). The outflowing plasma is an example of fast adiabatic expansion, as described in the previous section. The resulting emission spectrum is a superposition of delayed recombination and line emission. I emphasize this conjunction, because on the basis of an energy ar- 
gument the significance of this process has been challenged by Don Cox (see this volume). It is true that the contribution of recombination can be low in the case of a galactic wind with a disk averaged mass loss rate of $0.4 \mathrm{M}_{\odot} / \mathrm{yr}$ (Breitschwerdt and Schmutzler, 1994), although depending strongly on the initial conditions chosen. However, looking along the axis of the flow, e.g. in the direction of the North Galactic Pole from beneath the disk, we sample line emission from gas at different "temperatures", which is always out of equilibrium. This is fundamentally different from all hydrostatic, isothermal halo models. There is circumstantial evidence for this to have happened in the case of the soft X-ray emission in the halo of the edge-on galaxy NGC 4631. Wang et al. (1995) have reported that it is not possible to fit the halo spectrum by a single temperature of a plasma in CIE.

Another important key observation which may help us in our understanding of the Local Bubble concerns other superbubbles and in particular our nearest neighbour Loop I or North Polar Spur. On the basis of ROSAT PSPC observations, it has been shown (Egger, 1993; Egger and Aschenbach, 1995) that Loop $I$ is an active superbubble with a present temperature of $4 \times 10^{6} \mathrm{~K}$ and a density of $2.5 \times 10^{-3} \mathrm{~cm}^{-3}$. The dense wall at a distance of $40 \pm 25 \mathrm{pc}$ as inferred from optical and UV spectral analysis of stars is most likely the interaction zone between Loop I and the Local Bubble. It can be shown (Breitschwerdt and Egger, 1997) that due to the overpressure of the plasma in Loop I, it is possible to excite a hydromagnetic Rayleigh-Taylor instability even in the presence of a magnetic field parallel to the interaction shell (by the way, this was also pointed out by Franz Kahn in the plenary discussion). The typical growth time of the most unstable mode is $\tau_{g} \approx \sqrt{8 \pi / n_{\mathrm{sh}}} N_{\mathrm{sh}} B_{0} \cos \alpha / \Delta P \approx 2.3 \times 10^{6} \mathrm{yr}$, where $n_{\mathrm{sh}}=15 \mathrm{~cm}^{-3}, B_{0}$ and $\Delta P=1.4 \times 10^{-12} \mathrm{~g} /\left(\mathrm{cm}^{2} \mathrm{~s}\right)$ are the density of the shell with $N_{\mathrm{sh}}=\int_{0}^{L} n_{\mathrm{sh}} d l=10^{20} \mathrm{~cm}^{-2}$ being its column density (and $L$ is the distance to the wall plus the shell thickness), the magnetic field strength and the pressure difference between Loop I and the Local Bubble, respectively; $\alpha$ is the angle between the direction of the magnetic field and the linear perturbation wave vector, with $B_{0} \cdot \cos \alpha$ taken to be $1 \mu \mathrm{G}$. It turns out that the most unstable wavelength is about $3 \mathrm{pc}$, i.e. similar to the shell thickness. After the full fledged instability has developed and the shell starts to fragment, blobs of this size will travel ballistically through the tenuous medium of the Local Bubble. A simple calculation shows that the velocity is given to be $v_{\mathrm{bl}}=3 \Delta P \tau_{g} /\left(\vec{m} N_{\mathrm{sh}}\right) \approx 22 \mathrm{~km} / \mathrm{s}$. This value is fairly close to the observed velocity vector of $26 \mathrm{~km} / \mathrm{s}$ of the LIC cloud (see Lallement this volume).

Far from being a direct proof, I am nevertheless tempted to conclude that the Local Bubble is a separate and rather common bubble that undergoes interaction with an other bubble; such a process might be more frequent than one would commonly think of, because star forming regions are not homogeneously distributed over the galactic disk. The presence of clouds in bubbles would then be simply a consequence of an inherent dynamic instability. 


\section{Open Questions}

Rather than giving any conclusions here, I would like to end with a list of questions about the LISM and its relation to the general ISM, which I had written down when I was organizing the scientific part of this conference.

1. What is the geometry of the Local Bubble?

2. What is the state of the plasma inside?

3. How do clouds and HI filaments in the Local Bubble survive despite their much lower apparent thermal pressure?

4. Is the Local Bubble magnetized and if so, what is the topology of the magnetic field?

5 . What is the origin of the Local Bubble?

6. What do cosmic rays tell us about nearby explosive events?

7. How does the Local Bubble connect to its environment?

8. How does the Local Bubble compare to other bubbles/superbubbles?

9. How does the LISM relate to the general ISM?

10. What is the origin of soft X-ray emission outside bubbles/superbubbles in the disk and halo?

11. What can we learn from the study of the ISM in other galaxies?

12. What are the future prospects for observation and theory?

In the previous sections I have sketched my view on some possible solutions, but now it is time to let the experts give their observational facts and interpretations on the "Local Bubble and Beyond".

Acknowledgements. I thank all participants for excellent contributions and lively and stimulating discussions during the meeting. I benefitted from many useful talks with my colleagues Roland Egger and Michael Freyberg. I thank Stu Bowyer and Chris McKee for helpful comments on the manuscript. Financial support from the DFG by a Heisenberg Fellowship is gratefully acknowledged.

\section{References}

Adams, W.S. (1948): ApJ 109, 354

Bowyer, C.S., Field, G.B., Mack, J.E. (1968): Nature 217, 3

Bregman, J.N. (1980): ApJ 236, 577

Breitschwerdt, D. (1996): Sp. Sci. Rev. 78, 173

Breitschwerdt, D. (1994): Habilitationsschrift, Universität Heidelberg, 158p.

Breitschwerdt, D., Egger, R.J. (1997): A\&A (in preparation)

Breitschwerdt, D., Schmutzler, T. (1997): A\&A (to be submitted)

Breitschwerdt, D., Schmutzler, T. (1994): Nature 371, 774

Breitschwerdt, D., McKenzie, J.F., Völk, H.J. (1993): A\&A 269, 54

Breitschwerdt, D., McKenzie, J.F., Völk, H.J. (1991): A\&A 245, 79

Brinks, E., Shane, W.W. (1984): ApJS 55, 179

Burrows, D.N., Mendenhall, J.A. (1991): Nature 351, 629 
Cox, D. P., Anderson, P.R. (1982): ApJ 253, 268

Cox, D.P., Reynolds, R.J. (1987): ARA\&A 25, 303

Cox, D.P., Smith, B.W. (1974): ApJ 189, L105

Egger, R.J. (1993): PhD Thesis, TU München, MPE Report 249, 111p.

Egger, R.J., Aschenbach, B. (1995): A\&A 294, L25

Field, G.B., Goldsmith, D.W., Habing, H.J. (1969): ApJ 155, L14

Fruscione, A., Hawkins, I., Jelinsky, P., Wiecigroch, A. (1994): ApJS 94, 127

Gwinn, C.R., Taylor, J.H., Weisberg, J.M., Rawley, L.A. (1986): AJ 91, 338

Hartman, J. (1904): in Sitzb. Kgl. Akad. Wiss. 527, translated in ApJ 19, 268

Heiles, C. (1980): ApJ 235, 833

Herbstmeier, U., et al. (1995): A\&A 298, 606

Ikeuchi, S. (1988): Fund. Cosmic Phys. 12, 255

Innes, D.E., Hartquist, T.W. (1984): MNRAS 209, 7

Jelinsky, P., Vallerga, J.V., Edelstein, J. (1995): ApJ 442, 653

Jenkins, E.B., Meloy, D.A. (1974): ApJ 193, L12

Kahn, F.D. (1976): A\&A 50, 145

Kahn, F.D., (1981): in Investigating the Universe, ed. F.D. Kahn, Reidel, 1

Kerp, J., Herbstmeier, U., Mebold, U. (1993): A\&A 268, L2

Linsky, J. (1996): Sp. Sci. Rev. 78, 157

McCammon, D., Sanders, W.T. (1990): ARA\&A 28, 657

McKee, C.F., Ostriker, J.P. (1977): ApJ 218, 148

Norman, C.A., Ikeuchi, S. (1989): ApJ 345, 372

Pikel'ner, S. (1967): Astr. Zu. 44, 915

Plaskett, J.S., Pearce, J.A. (1933): Publ. Dom. Astr. Obs. Victoria, B.C. 5, 167

Raymond, J.C., Smith, B.W.: (1977) ApJS 35, 419

Reynolds, R. (1991): in The interstellar disk-halo connection in galaxies, ed. $\mathrm{H}$. Bloemen, Reidel, 67

Sanders, W.T. (1993): Adv. Space Res. 13, (12)77

Sanders, W.T., Kraushaar, W.L., Nousek, J.A., Fried, P.M. (1977): ApJ 217, L87

Schmitt, J.H.M.M., Snowden, S.L. (1990): ApJ 361, 207

Snowden, S.L. (1996): MPE-Report 263, 299

Snowden, S.L., Cox, D.P., McCammon, D., Sanders, W.T. (1990): ApJ 354, 211

Snowden, S.L., et al. (1991): Science 252, 1529

Snowden, S.L., McCammon, D., Verter, F. (1993): ApJ 409, L21

Sonett, C.P., Morfill, G.E., Jokipii, J.R. (1987): Nature 330, 458

Spitzer, L. jr. (1956): ApJ 124, 20

Strömgren, B. (1939): ApJ 89, 526

Tanaka, Y., Bleeker, J.A.M. (1977): Sp. Sci. Rev. 20, 815

Tauris, T.M., et al. (1994): ApJ 428, L53

Taylor, J.H., Cordes, J.M. (1993): ApJ 411, 674

Trümper, J. (1983): Adv. Sp. Res. 2(4), 241

van de Hulst, H.C. (1945): Ned. Tijdschr. Natuurk. 11, 201

Wang, Q.D., et al. (1995): ApJ 439, 176

York, D.G. (1974): ApJ 193, L127 